\title{
Acoso sexual callejero, ¿no es para tanto o es para mucho? Percepciones sobre la violencia contra las mujeres en Costa Rica
}

\author{
Street sexual harassment, is not it for so much or is it \\ for a lot? Perceptions about violence against women in \\ Costa Rica
}

Laura Solís Bastos Instituto de Estudios Sociales en Población (IDESPO)

Universidad Nacional, Costa Rica laura.solis.bastos@una.cr

\begin{abstract}
Resumen
El acoso sexual callejero es una de las formas de violencia contra las mujeres más común en América Latina. A través de los resultados de una encuesta telefónica realizada a nivel nacional por el Instituto de Estudios Sociales en Población (IDESPO), fue posible identificar las percepciones de la población sobre acoso sexual callejero y violación de derechos humanos en Costa Rica.

Palabras claves: acoso sexual callejero, derechos humanos, género, violencia.

Abstract

Street sexual harassment is one of the most common forms of violence against women in Latin America, through the results of a telephone survey conducted at the national level by the Institute of Social Studies in Population (IDESPO), it was possible to identify the perceptions of the population about street sexual harassment and violation of human rights in Costa Rica.
\end{abstract}

Keywords: street sexual harassment, Human rights, gender, violence 


\section{Introducción}

$\mathbf{E}$ 1 acoso sexual callejero ha sido denominado como una de las formas más comunes de violencia contra las mujeres en América Latina; sin embargo, solapado tras supuestos elementos culturales ha tendido a ser una conducta naturalizada, lo cual ha permitido cierto rango de permisividad y aceptación social.

Este fenómeno social generalmente ha sido estudiado desde la contabilización y generación de estadísticas referentes a los incidentes ocurridos, donde lo común es dar a conocer cuántas mujeres han sido víctimas de este tipo de violencia y de qué formas; ante esto, surge el interés de estudiar este fenómeno, pero desde otra perspectiva, desde las percepciones, específicamente desde un enfoque de derechos humanos.

Es así como el interés se enfoca en analizar los datos obtenidos en la encuesta realizada por el Programa Umbral Político del Instituto de Estudios Sociales en Población (IDESPO), de la Universidad Nacional de Costa Rica, para valorar las percepciones de la población costarricense sobre el acoso sexual callejero y cuánto consideran que estas diversas manifestaciones son o no violaciones de los derechos humanos.

Si bien, el acoso callejero pareciera no ser "una nueva forma de violencia", su reconocimiento como tal sí lo es; aunado a esto surgen una serie de resistencias de forma más reciente respecto a las herramientas judiciales que penan estos hechos; lo anterior, en el marco de las discusiones que se desarrollan en espacios como medios de comunicación y redes sociales, en los que se emiten diversas opiniones ante conflictos que acontecen en la cotidianidad.

\section{Metodología}

Este artículo expone, de forma parcial, algunos de los resultados de la encuesta Percepciones sobre convivencia y derechos humanos en Costa Rica, la cual fue realizada por el Programa Umbral Político, del Instituto de Estudios Sociales en Población (IDESPO) de la Universidad Nacional de Costa Rica (UNA).

La encuesta se realizó mediante llamada telefónica a hogares que cuentan con servicio proporcionado por el Instituto Costarricense de Electricidad (ICE), se aplicó en dos periodos, del 13 al 26 de marzo y del 28 al 30 de abril del año 2017.

Se empleó una muestra, de 800 personas, mayores de edad, costarricenses o extranjeras con tres o más años de vivir en el país. La muestra se constituyó a partir de cuotas de sexo y edad. Así, el 52,13\% de la población entrevistada fue de sexo femenino y el 47,88\% fue de sexo masculino. Los resultados cuentan con un nivel de confianza del $95 \%$ y un margen de error de $\pm 3,5 \%$; lo cual garantiza que son representativos de las percepciones de la población costarricense sobre los temas consultados.

\section{Acoso sexual callejero un tema de derechos humanos}

El acoso sexual callejero ha sido un tema de coyuntura en los últimos años en el país, principalmente por hechos específicos que se han suscitado como referencia a nivel público; el más recordado lo constituye la 
denuncia realizada por el ciudadano Gerardo Cruz, a través de la publicación de un vídeo en Facebook, en el año 2015.

Es necesario destacar que el acoso sexual callejero debe ser catalogado como una práctica cultural y cotidiana, que no puede ser concebida como un hecho social que ha emergido de forma reciente; por el contrario, esta práctica en el pasado había sido naturalizada por la sociedad, por lo que no era reconocida como una conducta inapropiada.

Sin embargo, en el contexto actual, se debe recalcar que las percepciones han ido variando, en tanto la población costarricense tiende a reconocer que el acoso sexual callejero como una forma de violencia de la cual las principales víctimas son las mujeres.

Es necesario aclarar, a nivel conceptual, lo que se comprende por acoso sexual callejero para el caso costarricense, según el Observatorio de Violencia de Género contra las Mujeres y Acceso a la Justicia:
Por acoso callejero se entiende cualquier conducta con connotación sexual explícita o implícita, proviene de un desconocido, ocurrido en espacios públicos y tiene el potencial de provocar malestar en el o la acosada. Es un tipo de violencia por ser una práctica no deseada, que genera un impacto psicológico negativo en las personas, especialmente mujeres, algunas desde los 12 años, en promedio, quienes pueden ser víctimas de acoso callejero varias veces al día. (Observatorio de violencia de género contra las mujeres y acceso a la justicia, s. f. párr.1)

Durante el año 2016, se procedió con un total de 6489 denuncias vinculadas a distintas manifestaciones de acoso sexual callejero en el país, las cuales fueron presentadas ante los juzgados contravencionales; sin embargo, el número de hechos puede ascender a una cifra mayor en el tanto muchas personas no llegan a presentar este
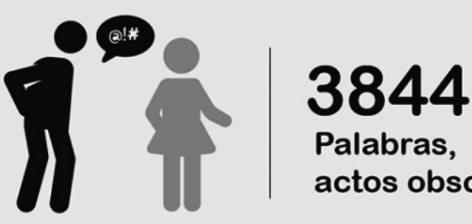

Palabras, actos obscenos

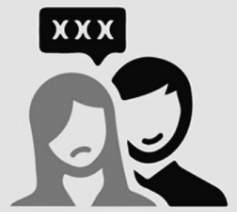

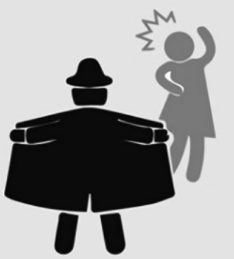

188

Exhibicionismos

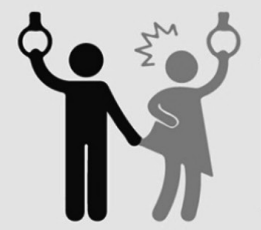

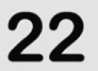

Tocamientos

Figura 1. Denuncias de acoso sexual callejero en el año 2016

Nota: Datos preliminares de casos denunciados ante los Juzgados Contravencionales en el año 2016, según la Sección de Estadística del Departamento de Planificación del Poder Judicial de Costa Rica, disponibles en la página web del Observatorio de Violencia de Género contra las Mujeres y Acceso a la Justicia. 
tipo de denuncias, por temor, vergüenza, e impotencia, entre otras razones.

Se concibe el concepto de derecho humano como se estipula en la Declaración Universal de Derechos Humanos emitida en 1948.

Artículo 1: Todos los seres humanos nacen libres e iguales en dignidad y derechos $\mathrm{y}$, dotados como están de razón y conciencia, deben comportarse fraternalmente los unos con los otros.

Sin embargo, a pesar del avance en el alcance y respecto a los derechos humanos, "el acoso sexual callejero tiene el potencial de impactar en la percepción de seguridad en lugares públicos y en limitar su libertad de movimiento y acceso a éste" (Llerena, 2016, p. 64).

Por otra parte, persisten de forma simbólica relaciones de dominación, explotación y conflicto entre actores sociales en el marco de la colonialidad, tal como lo ha planteado Quijano (2001, citado por Quintero, 2010), pues, de esta, el género no se excluye, pues la sociedad se ha construido desde una visión patriarcal y heterosexual en la cual se naturalizaron diversas formas de violencia contra las mujeres.

Es así como el acoso callejero, desde esta perspectiva, se visualiza como una forma de violencia contra la mujer: "Mientras que el acoso callejero es usualmente visto como benigno o poco dañino. Estudios postulan que esas experiencias pueden generar efectos negativos profundos en las víctimas" (Llerena, 2016, p. 64).

De acuerdo con los resultados obtenidos en la encuesta, fue posible el reconocimiento de distintas formas de acoso sexual callejero, pero además el objetivo principal de estas interrogantes se dirigió a identificar si la población costarricense encuestada concibe estas conductas de violencia sexual como una forma de violación de los derechos humanos de las personas.

Tabla 1. Acoso sexual callejero. ¿Cuánto considera usted que estas situaciones son violaciones a los derechos humanos? $n=800$

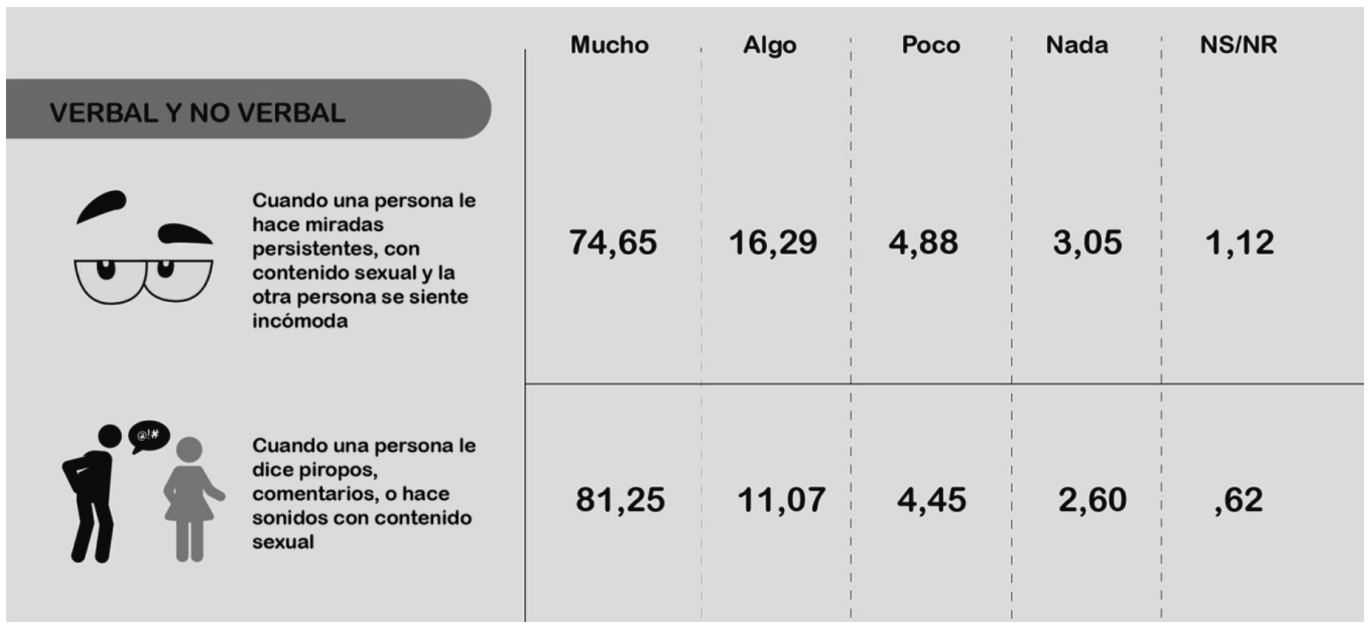



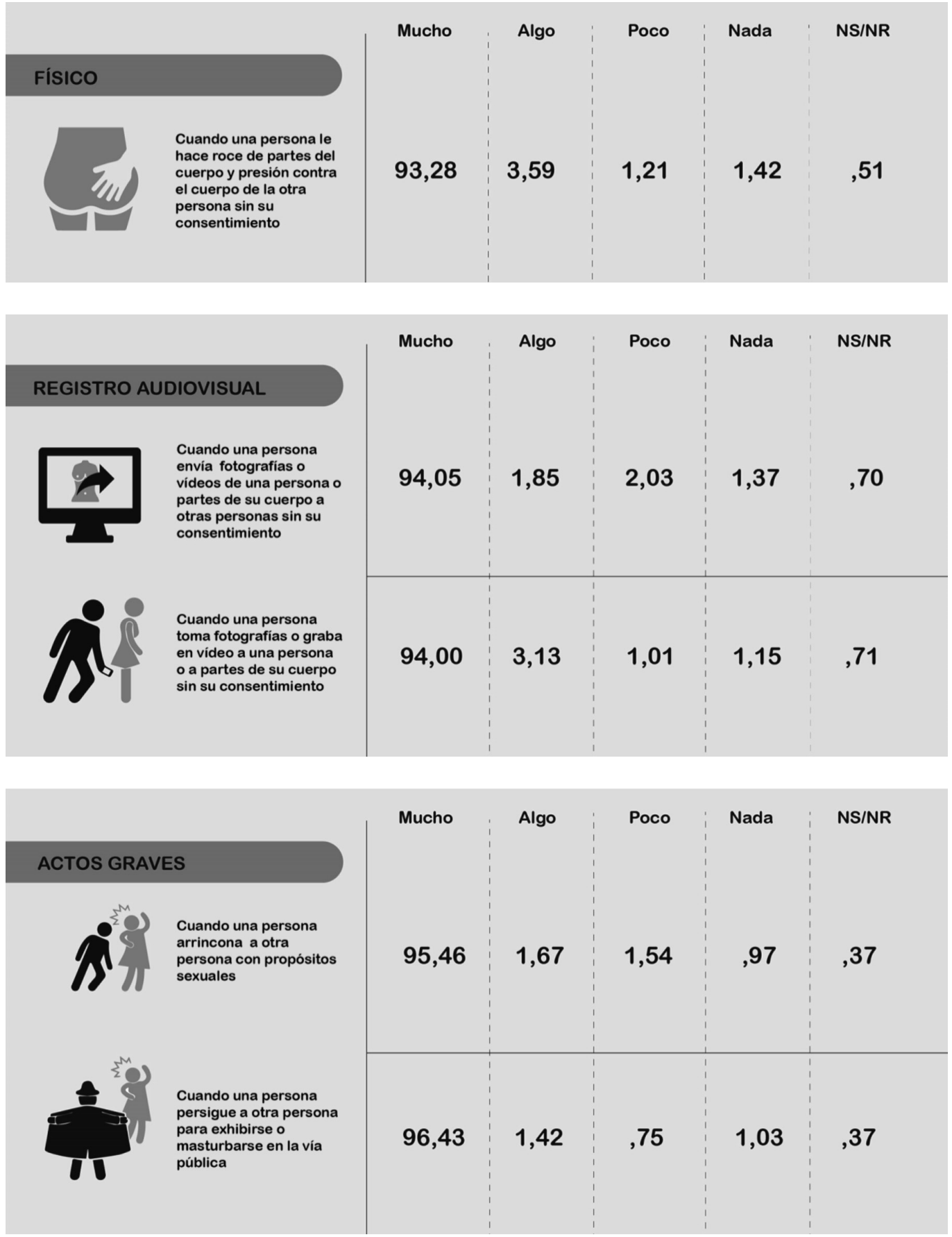

Nota: IDESPO-UNA. Encuesta Percepción sobre aspectos de los derechos de las personas en Costa Rica, 2017. 
En los resultados obtenidos se destaca que una gran mayoría de la población encuestada observa, en las situaciones descritas de acoso sexual callejero, manifestaciones de violación de los derechos humanos; sin embargo, debe resaltarse que los ejemplos descritos se asociaron a cuatro tipos de violencia presentes como la verbal y no verbal, la física, el registro audiovisual y actos graves. De ello se destaca que en los resultados obtenidos, para la categoría "mucho" de la escala de valoración propuesta, existe una tendencia a calificar a algunas de estas situaciones con un porcentaje mayor, tal como se muestra en la siguiente figura:

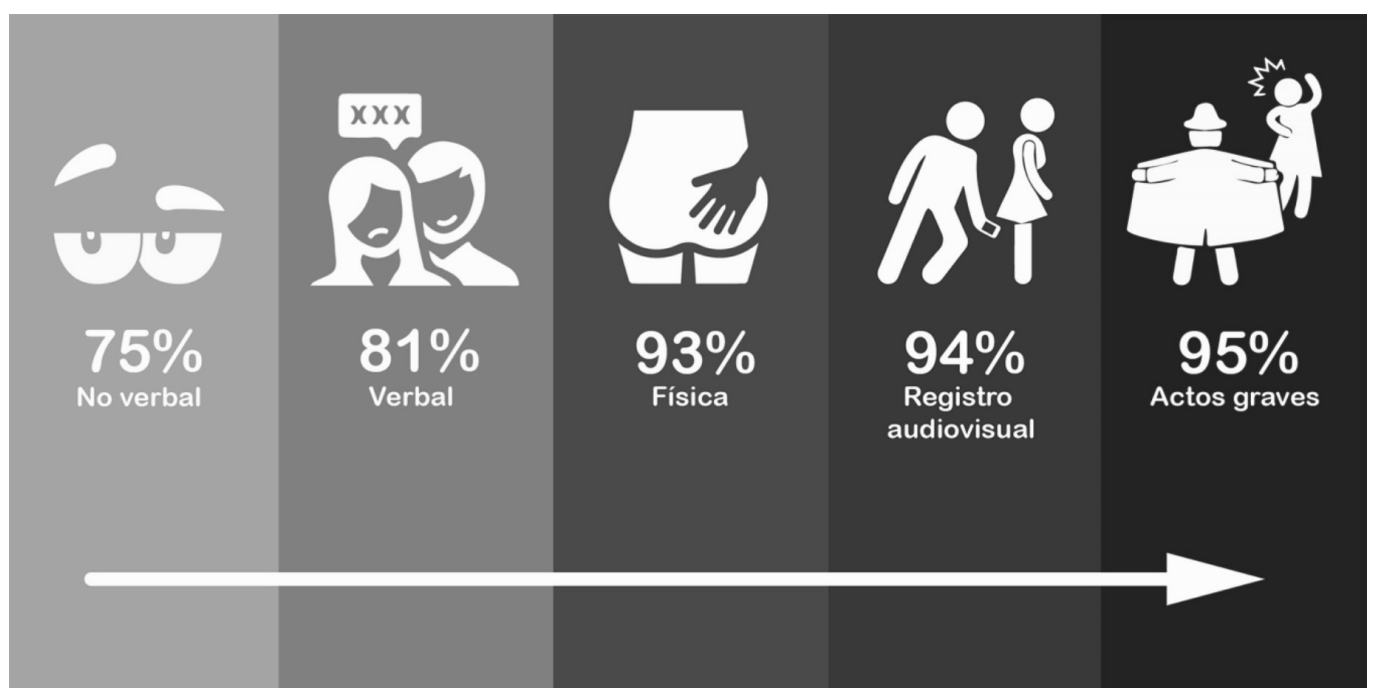

Figura 2. Representación porcentual del acoso sexual callejero como una violación de los derechos humanos, según tipo de manifestación de violencia.

Nota: IDESPO-UNA. Encuesta Percepción sobre aspectos de los derechos de las personas en Costa Rica, 2017.

De acuerdo con lo anterior, se observa una tendencia a calificar algunas situaciones más cercanas a ser consideradas como violación de derechos humanos, mientras que para otros casos se muestran más lejanas; esto es acorde con el nivel de calificación de lo que se consideraría como manifestaciones de mayor gravedad; ante lo cual debe aclararse que se hace hincapié en que todo acto asociado al acoso sexual callejero, sin diferencia de tipo de violencia, debe ser reconocido como una violación a los derechos humanos de las personas.
Ante esto, el acoso sexual callejero debe ser identificado como conductas improcedentes e inaceptables asociadas a la violencia de género, que van en contra de la dignidad de las personas al atentar contra derechos humanos como la integridad física, psicológica, el libre tránsito, principalmente.

En Costa Rica, el acoso sexual callejero no es considerado aún un delito como tal, dado que no existe una ley específica que permita sancionarlo; sin embargo, indirectamente pueden ser empleados otros 
mecanismos existentes para su sanción, como la contravención de palabras o actos obscenos contemplada en el artículo 392 del Código Penal.

Ante este vacío existente en el marco de una legislación específica para sancionar el acoso sexual callejero como delito, el reconocimiento de los derechos de las víctimas frente a esta situación y la evidencia de la presencia de estos hechos en la cotidianidad, se consultó a la población encuestada: ¿Considera usted que debería aprobarse una ley que sancione el acoso sexual callejero?

Tabla 2. Distribución porcentual de la población encuestada respecto a la consideración de aprobar una ley que sancione el acoso sexual callejero en Costa Rica. n=800
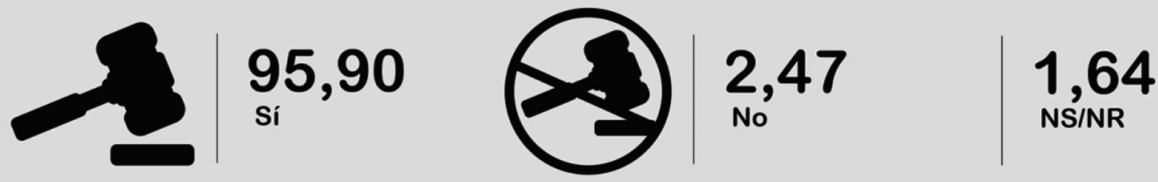

Fuente: IDESPO-UNA. Encuesta Percepción sobre aspectos de los derechos de las personas en Costa Rica, 2017.

Los datos anteriores evidencian un claro apoyo de parte de la población costarricense a la aprobación de una ley específica que sancione el acoso sexual callejero. Actualmente, dentro de la corriente legislativa se encuentra el proyecto "Ley contra el acoso sexual callejero", Expediente $N^{\circ}$ 20.299, el cual ha sido trasladado para el estudio e informe de la Comisión Permanente Especial de la Mujer.

\section{Consideraciones finales}

- El acoso sexual callejero suele ser una práctica de suma frecuencia que debe ser reconocida en todas sus formas como violencia que atenta contra los derechos humanos.
- Frente a estos actos, las mujeres son las principales víctimas de esta forma de violencia, por lo cual es, a su vez, una forma de violencia de género.

- Los datos de la encuesta presentan una ruptura epistemológica a partir del reconocimiento de que el acoso sexual callejero es percibido por la mayoría de las personas encuestadas como una violación de los derechos humanos de las personas.

- Costa Rica es reconocido a nivel internacional desde el punto de vista político por ser abanderado de los derechos humanos, razón por la cual el acoso sexual callejero debe ser un tema de interés desde el punto de vista 
legislativo y punitivo, que garantice los derechos humanos de las víctimas de este tipo de violencia, principalmente de las mujeres y, sobre todo, de aquellas que se encuentran en condiciones de mayor vulnerabilidad.

\section{Referencias}

Asamblea Legislativa Costa Rica. (2017). proyecto de Ley "Ley contra el acoso sexual callejero", Expediente $N .^{\circ}$ 20.299. Disponible en la página web http://www.poder-judicial.go.cr/ observatoriodegenero/wp-content/ uploads/2017/06/PROYECTO-DELEY-LEY-CONTRA-EL-ACOSOSEXUAL-CALLEJERO.pdf

IDESPO-UNA. (2017) Base de datos Encuesta Percepción sobre aspectos de los derechos de las personas en Costa Rica, Programa Umbral Político.

Llerena Benites, R. C. (2016). Percepción $\mathrm{y}$ actitudes frente al acoso sexual callejero en estudiantes mujeres de una universidad privada de Medicina. Revista Horizonte Médico, 16(1), 62-68. Disponible en http:// www.horizontemedicina.usmp.edu. pe/index.php/horizontemed/article/ view/397
Observatorio de Violencia de Género contra las Mujeres y Acceso a la Justicia. (2017). Datos preliminares casos denunciados ante los Juzgados Contravencionales en el año 2016, Sección de Estadística del Departamento de Planificación del Poder Judicial de Costa Rica. Disponible en http://www.poder-judicial.go.cr/observatoriodegenero/soy-mujer-y-busco/ averiguar-sobre-acoso-callejero/

Quintero, Pablo. (2010). Notas sobre la teoría de la colonialidad del poder y la estructuración de la sociedad en América Latina (Papeles de trabajo). Centro de Estudios Interdisciplinarios en Etnolingüística y Antropología Socio-Cultural, 19. Disponible en http://www.scielo.org.ar/scielo.php?script $=$ sci a r t t e x t \& p id = S 1852 $45082010000100001 \& \operatorname{lng}=$ es\&tln $\mathrm{g}=\mathrm{es}$ 